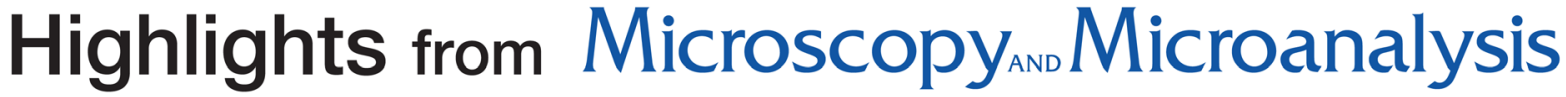

\section{Biological Applications}

Targeting Functionally Characterized Synaptic Architecture Using Inherent Fiducials and 3D Correlative Microscopy by CI Thomas, MA Ryan, B Scholl, D Guerrero-Given, D Fitzpatrick, and N Kamasawa, Microsc Microanal | doi: 10.1017/S1431927620024757

We established an EM-label-free correlative microscopy workflow to pair synaptic function with ultrastructural properties in cortical brain tissue. $\mathrm{Ca}^{2+}$ activity in neurons was captured using GCaMP6s and in vivo 2-photon light microscopy (LM), providing insight into their functional properties during visual stimulation. Conversely, volumetric electron microscopy (EM) provided detailed ultrastructural information of the neuron and subcellular features (that is, dendrites and dendritic spines). We used inherent fiducials, such as blood vessels and cell bodies in the tissue, to correlate: 1) in vivo 2-photon LM, 2) ex vivo confocal LM (that is, after fixing and slicing the brain), and 3) serial block face scanning EM. To improve image quality in our correlative workflow for brain tissue, we precisely optimized the sample preparation protocol for visualizing synaptic structures, and we applied improved electron detection hardware in the SEM. 3D EM reconstructions of the neuron could then be mapped back to in vivo functional data, allowing us to analyze functional properties of individual dendritic spines in the context of their structural characteristics (Figure).
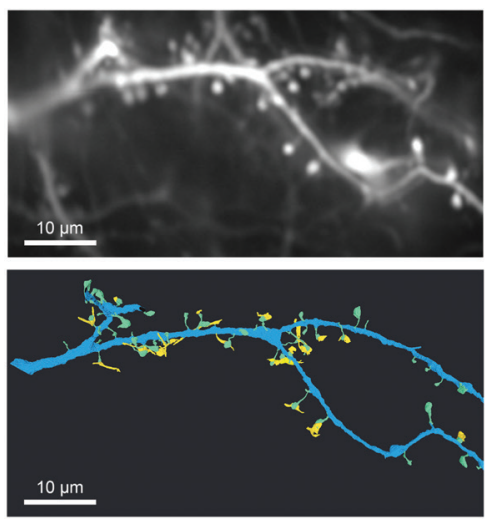

(Top) 2-photon image of a dendritic segment of a cortical pyramidal neuron, imaged using the $\mathrm{Ca}^{2+}$ indicator GCaMP6s. Along the dendrite are numerous dendritic spines, which are the sites of excitatory synapses onto the neuron. (Bottom) A 3D reconstruction of the same dendrite from SEM data, with the dendrite in blue, spines in green, and presynaptic axon segments in yellow. Correlative matching allows identification and paired function-structure measurements of individual dendritic spines.

\section{Materials Applications}

In situ TEM Characterization of Microstructure Evolution and Mechanical Behavior of the 3D-Printed Inconel 718 Exposed to High Temperature by S Koul, LZhou, O Ahmed, Y Sohn, T Jiang, and A Kushima, Microsc Microanal| doi: 10.1017/S1431927621000052

Understanding the effect of heat treatment on microstructure evolution and mechanical property is important for performing postproduction heat treatment of alloys produced by additive manufacturing processes. We performed in situ transmission electron microscopy (TEM) characterization of microstructural evolution in 3D-printed Inconel 718 (IN718) while exposed to elevated temperature and analyzed an associated change in mechanical property. A specially designed specimen shape that enables tensile testing of nano-sized thin films without off-plane deformations was used. Additionally, it allowed seamless transition between in situ heating and tensile experiments, using the same specimen, to elucidate direct correlation of microstructure evolution and mechanical property. A clear transition of the failure mode from ductile to brittle was observed after exposing the as-printed IN718 to high temperature, where the residual stress relaxation and the formation of incoherent $\gamma^{\prime}$ precipitates took place (Figure). Ductility was maintained with a full heat treatment with coherent $\gamma^{\prime}$ precipitates.

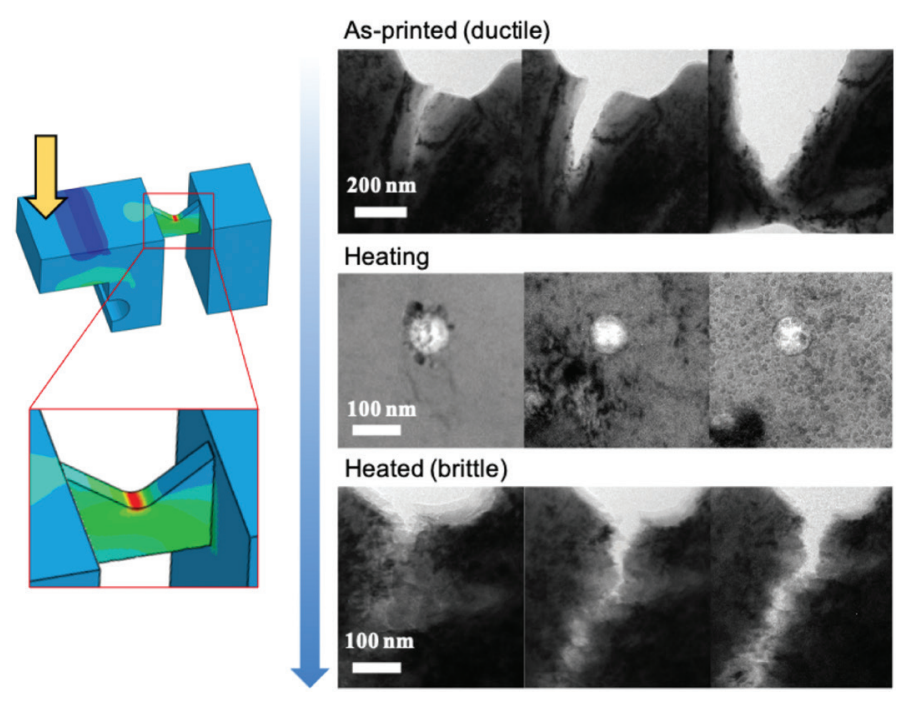

Push-to-pull specimen design that prevents off-plane deformation and allows seamless transition from heating to tensile experiment inside a TEM. Failure mode of the as-printed IN718 changed from ductile to brittle after direct heating. 


\section{Microscopy Microanalysis}

\section{Techniques Development}

Integrative Atom Probe Tomography using STEM-Centric Atom Placement as a Step Toward Atomic-Scale Tomography by AV Ceguerra, AJ Breen, JM Cairney, SP Ringer, and BP Gorman, Microsc Microanal | doi.org/10.1017/S1431927620024873

Current $3 \mathrm{D}$ atom position reconstruction methodologies for atom probe tomography (APT) contain serious geometric artifacts that are difficult to address, due to their reliance on empirical factors to generate a volume. To overcome this limitation, a reconstruction technique is demonstrated where the analyzed volume is instead defined by the specimen geometry and crystal structure, as determined by transmission electron microscopy (TEM) and diffraction acquired before and after APT analysis. APT data are reconstructed using a bottom-up approach, where the post-APT TEM image is used to define the substrate upon which APT detection events are placed (Figure). Transmission electron diffraction enables the quantification of the relationship between atomic positions and the evaporated specimen volume. Using an example dataset of $\mathrm{ZnMgO}$ :Ga grown epitaxially on c-plane sapphire, a volume is reconstructed that has the correct geometry and atomic spacings in $3 \mathrm{D}$. APT data are thus reconstructed in $3 \mathrm{D}$, without using empirical parameters for the reverse projection reconstruction algorithm.

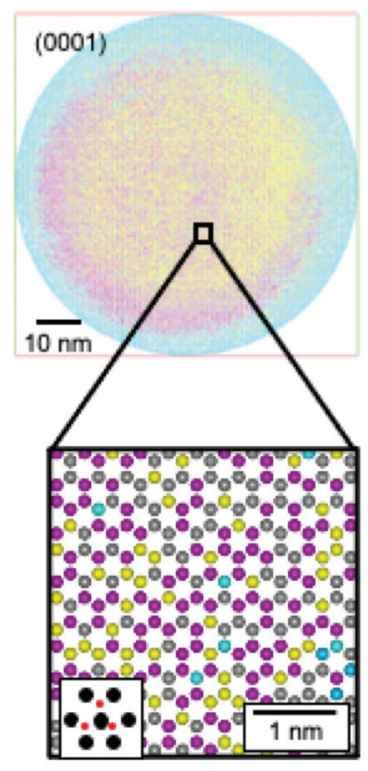

APT data reconstructed using TEM-defined atom positions. TEM imaging and diffraction are used in order to produce a specimen function consisting of the analyzed volume, crystallographic space group and orientation relative to the APT analysis directions, lattice spacings, and microstructural features.

\section{A top journal in Microscopy}

\section{Published for the Microscopy Society of America}

Editor: John Mansfield, University of Michigan, USA

The only journal owned by scientists and published for scientists, Microscopy and Microanalysis provides original research papers in the fields of microscopy, imaging and compositional analysis. This distinguished international forum is intended for microscopists in both biology and materials science.

\section{Online submission at cambridge.org/mam/submit}

View the journal online at cambridge.org/mam

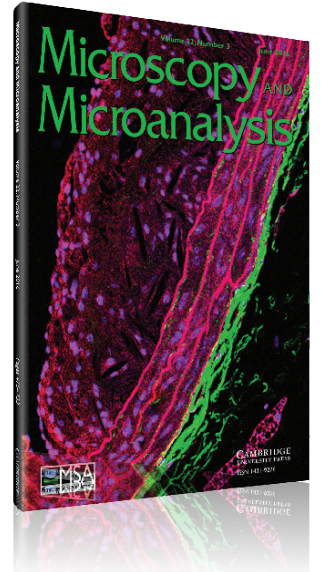

CAMBRIDGE

UNIVERSITY PRESS 